\title{
Predicting trajectories of behavioral adjustment in children diagnosed with acute lymphoblastic leukemia
}

\author{
Simone M. Sint Nicolaas ${ }^{1} \cdot$ Peter M. Hoogerbrugge ${ }^{2,3,4} \cdot$ Esther M. M. van den Bergh $^{4}$. \\ José A. E. Custers $^{1} \cdot$ Sofia Gameiro $^{5} \cdot$ Reinoud J. B. J. Gemke ${ }^{6} \cdot$ Chris M. Verhaak $^{1}$
}

Received: 10 February 2016 / Accepted: 30 May 2016 /Published online: 14 June 2016

(C) The Author(s) 2016. This article is published with open access at Springerlink.com

\begin{abstract}
Purpose Previous research showed that children with cancer are at risk for developing behavioral adjustment problems after successful treatment; however, the course of adjustment remains unclear. This study focuses on adjustment trajectories of children during treatment for acute lymphoblastic leukemia (ALL) and aims to distinguish subgroups of patients showing different trajectories during active treatment, and to identify sociodemographic, medical, and psychosocial predictors of the distinct adjustment trajectories.

Methods In a multicenter longitudinal study, 108 parents of a child (response rate $80 \%$ ) diagnosed with ALL were assessed during induction treatment (T0), after induction/consolidation treatment (T1), and after end of treatment (T2). Trajectories of
\end{abstract}

Electronic supplementary material The online version of this article (doi:10.1007/s00520-016-3289-9) contains supplementary material, which is available to authorized users.

Simone M. Sint Nicolaas

simone.sintnicolaas@radboudumc.nl

1 Department of Medical Psychology 840, Radboud University Nijmegen Medical Center, PO Box 9101, 6500

HB Nijmegen, The Netherlands

2 Pediatric Oncology, Radboud University Medical Center, Nijmegen, The Netherlands

3 Dutch Childhood Oncology Group (SKION), The Hague, The Netherlands

4 Princess Máxima Center for Pediatric Oncology, Utrecht, the Netherlands

5 Cardiff Fertility Studies Research Group, School of Psychology, Cardiff University, Cardiff, Wales, UK

6 Pediatrics, VU University Medical Center. Amsterdam, The Netherlands child behavioral adjustment (Child Behavior Checklist; CBCL) were tested with latent class growth modeling (LCGM) analyses.

Results For internalizing behavior, a three-trajectory model was found: a group that experienced no problems $(60 \%)$, a group that experienced only initial problems $(30 \%)$, and a group that experienced chronic problems $(10 \%)$. For externalizing behavior, a three-trajectory model was also found: a group that experienced no problems (83\%), a group that experienced chronic problems (12\%), and a group that experienced increasing problems ( $5 \%)$. Only parenting stress and baseline QoL (cancer related) were found to contribute uniquely to adjustment trajectories.

Conclusions The majority of the children ( $77 \%$ ) showed no or transient behavioral problems during the entire treatment as reported by parents. A substantial group (23\%) shows maladaptive trajectories of internalizing behavioral problems and/ or externalizing behavioral problems. Screening for risk factors for developing problems might be helpful in early identification of these children.

Keywords Pediatric oncology $\cdot$ ALL $\cdot$ Behavioral adjustment $\cdot$ Trajectories $\cdot$ Predictors

\section{Introduction}

Acute lymphoblastic leukemia (ALL) is the most common form of childhood cancer and is characterized by a mainly young age at diagnosis, long duration of treatment, and severe family burden [1]. In the long term, children diagnosed with leukemia are at particularly high risk for behavioral and neuropsychological difficulties, partly due to their intensive therapy $[2,3]$. Retrospective studies have shown that up to $60 \%$ of the patients treated for childhood ALL report impairments 
in various neurocognitive domains [4]. As these impairments are often ongoing and significantly impact the quality of life (QoL) in cancer survivors, long-term monitoring of psychological functioning is needed $[3,5]$.

Although it seems clear that childhood cancer survivors are at risk for psychological problems, the link between adaptation during treatment and long-term difficulties is not clear yet. Previous studies showed that children in treatment adapt adequately when analyzed as a group: some studies found increased levels of problems shortly after diagnosis, which normalized during treatment $[6,7]$, while other studies report no behavioral problems in children with cancer [8]. Most longitudinal studies have investigated adaptation over time at group level, which might mask individual differences. It is likely that children with cancer do not show a single pattern of adjustment over time, and therefore, the investigation of individual trajectories is important $[9,10]$. Studies describing the longitudinal trajectories of adaptation are scarce. It is possible that the adaptation in children diagnosed with ALL can also be classified into different trajectories, such as documented before in adult care [9-11]. No research has yet distinguished the trajectories of behavioral adjustment in pediatric oncology. It is important to start investigating behavioral adjustment problems in an early phase of treatment, because we know that this has substantial impact on adaptation on the long term [12]. Therefore, it is important to identify the trajectories of adjustment through the illness trajectory into long-term survivorship [9]. When distinct adjustment trajectories are found, predictors of these trajectories can be investigated. This information is relevant in early identification of children showing a maladaptive adjustment trajectory, and this is informative for how and when to target psychosocial services.

Previous research suggests that there are sociodemographic, medical, and psychosocial factors which are predictive of child maladjustment after cancer diagnosis. Studies showed that younger child age [13], lower parental education level [14], single-parent household [15], and hospitalizations [7] are all risk factors for child maladjustment. Next to this, a consistent link has been found between low levels of child adaptation response immediately after diagnosis and later adjustment $[14,15]$. At last, parental psychosocial factors, including distress [16], low social support [17], helpless cognitions [18], avoidant/passive coping [19], and parenting stress [20,21] were consistently found to be risk factors for child maladjustment.

The first aim of the present study was to identify distinct subgroups of patients diagnosed with ALL showing different adjustment trajectories during active treatment. The second aim of this study was to explore the demographic, medical, and psychosocial predictors of these adjustment trajectories. In line with previous literature, we hypothesized that worse child baseline response, parental negative affect, parental helpless cognitions, parenting stress, low parental social support, and low parental acceptance would be associated with maladaptive adjustment trajectories. Medical factors such as hospitalizations and ICU admissions were also expected to be related to adjustment trajectories.

\section{Methods}

\section{Sample}

Parents of children with ALL from six of seven Dutch pediatric oncology centers were enrolled. Inclusion criteria were (1) child age between 1.5 and 18 years, (2) newly diagnosed with ALL, and (3) treated according to the Dutch Childhood Oncology Group (DCOG) ALL 10 protocol [22]. In addition, parents had to be fluent in Dutch and children with an important pre-existing condition (e.g., Down syndrome), potentially affecting baseline measurement, were excluded.

\section{Procedure}

From October 2006 till October 2009, parents of newly ALLdiagnosed patients were invited to participate in this study. Parents who were willing to participate received verbal and written information on the study within the first weeks after diagnosis by one of the principal researchers. Families were instructed to choose one parent respondent for all assessments. Informed consent was obtained from all participants in this study. Parent-proxy measurements were performed three times for the children treated according to the standard-risk (SR) or medium-risk (MR) ALL-10 protocol: during induction treatment, after ending induction/consolidation treatment, and during maintenance, and shortly after finishing treatment. For children treated according to the HR ALL-10 protocol, measurements were performed only two times due to higher intensity and shorter duration of treatment: during induction treatment, and shortly after finishing treatment. The study was approved by each of the medical ethical review boards of the participating institutions.

\section{Measures}

Sociodemographic information (gender, date of birth, socioeconomic status, family situation, treatment protocol, number of hospitalizations, and number of ICU admissions) was collected with a self-developed questionnaire.

Parental subjective well-being was assessed with the Dutch shortened version of the Profile of Mood States (POMS) [23, 24]. The shortened POMS consists of 32 items and is designed to measure mood in five different domains. The answers are graded on a 5-point scale ranging from "not at all" (0) to "extremely" (4). Norm scores are available [23]. In the current study, internal consistency for the different domains ranged from $\alpha=.79$ to $\alpha=.91$. 
Parental illness cognitions were assessed with the Illness Cognitions Questionnaire - parent version (adapted from the ICQ) $[25,26]$. The ICQ-p measures illness cognitions that reflect different ways of evaluating the aversive character of a chronic condition of the child. In the current study, internal consistency for the subscales ranged from $\alpha=.75$ to $\alpha=.87$.

Parental coping strategies were assessed with the Utrecht Coping List (UCL) [27]. Two of the seven scales were used in the current study, namely the less adaptive coping strategy avoidance/awaiting (eight items) and passive reaction pattern (seven items). Items are scored on a 4-point scale. Internal consistency for the current sample was $\alpha=.47$ for the avoidance/awaiting subscale (this subscale was excluded from analyses due to limited reliability) and $\alpha=.64$ for the passive reaction pattern subscale.

Parental parenting stress was assessed with the Parenting Stress Index-short form (PSI) [28]. The PSI measures the level of stress parents experience in raising their child and it consists of 25 statements on a 6-point Likert scale. In the current study, the total stress score was used as a measure of parenting stress. In the current sample, internal consistency was $\alpha=.92$.

Parental perceived social support was assessed with the Inventory for Social Reliance (ISR) [29]. The subscale potential emotional support was used in the current study, which consists of five items measuring perceived social support. Internal consistency for the current sample was $\alpha=.92$.

Child generic quality of Life ( $Q o L)$ was assessed with the Child Health Questionnaire (CHQ) [30]. The CHQ is a 50item parent-reported questionnaire, covering the physical, emotional, and social well-being of children. Items are scored on a 4- to 6-item Likert scale and converted to a 0-100 score, with higher scores indicating higher QoL. Two summary scores are available (physical and psychosocial). Internal consistency for the total questionnaire in this sample was $\alpha=.69$.

Child disease-specific $Q o L$ was assessed with the PedsQL cancer module [31]. This is a 27-item multidimensional cancer-specific questionnaire. Items are scored on a 4-point Likert scale with a higher score indicative for better QoL. Internal consistency was $\alpha=.82$ for the total scale in the current sample.

Parent-rated child behavioral adjustment was collected using the Dutch translation of the Child Behavior Checklist $[32,33]$. The CBCL is a parent-reported questionnaire that provides scores on global, internalizing, and externalizing behavioral problems. In this study, two distinct versions were used, one for children aged 1.5-5 years (101 items), and one for 6-18 years (113 items). Items are scored on a 3-point Likert scale; a total problem score is obtained by summing item scores. Available norms provide age and genderstandardized T-scores $($ mean $=50 ; \mathrm{SD}=10)$ [32]. For analyses on trajectories, T-scores could not be used because they differed between the two age versions. Therefore, only items that appear on both age versions of the CBCL were used to include all in the same analysis [34, 35]. For the internalizing scale, six items were used ("Too fearful or anxious," "Self-conscious or easily embarrassed," "Shy or timid," "Unhappy, sad, or depressed," "Withdrawn, doesn't get involved with others," and "Worries"). These items correlated highly with the T-score of the internalizing scale $(r=78-.80)$ and showed acceptable reliability ( $\alpha=.77$ for CBCL version $1.5-5$ years and $\alpha=.74$ for CBCL version 6-18 years). Possible range for this scale was $0-12$ and mean score varied between 1.77 and 2.18 $(\mathrm{SD}=2.15-2.36)$. Based on a norm population of 2119 Dutch children, a cut-off score of $3(\mathrm{M}+1 \mathrm{SD} ; M=1.29, \mathrm{SD}=1.61)$ was defined to distinguish between children with and without clinically significant behavioral problems. For the externalizing scale, nine items were used ("Can't sit still, restless, or hyperactive," "Cruel to animals," "Destroys own things," "Destroys things belonging to family or others," "Disobedient," "Doesn't seem to feel guilty after misbehaving," "Gets in many fights," "Physically attacks people," and "Temper tantrums or hot tempered"). These items correlated highly with the T-score of the externalizing scale $(r=78-.82)$ and showed acceptable reliability $(\alpha=.81$ for CBCL version 1.5-5 years and $\alpha=.78$ for CBCL version 6 18 years). Possible range for this scale was 0-18 and mean score varied between 2.35 and $2.81(\mathrm{SD}=2.57-2.79)$. Based on the Dutch norm population, a cut-off score of 5 (M + 1SD; $M=2.22, \mathrm{SD}=2.35$ ) was defined to distinguish between children with and without clinically significant behavioral problems. During the study period, a total of 28 families switched from the CBCL 1.5-5 years to CBCL 6-18 years because aging of the child. When $50 \%$ or more of the items on a subscale were missing, the subscale score could not be computed and was handled as missing data. The CBCL has wellestablished reliability and validity [32].

\section{Statistical methods}

The Statistical Package for Social Sciences (SPSS) for Windows version 20.0 was used for descriptive analyses. Normal distribution of continuous data was assessed using skewness and kurtosis scores. All data showed a normal distribution. Trajectories of child behavioral adjustment were tested with latent class growth mixed modeling (LCGM) with maximum likelihood estimation using the R package LCMM [36].

We tested linear and quadratic models ranging from one to four trajectory groups. Multiple criteria were used for deciding which model (number and type of trajectories) better fits the data [37, 38]. First, we examined the Bayesian Information Criterion (BIC) and the Akaike Information Criterion (AIC). The closer the values are to 0 , the better the fit of the model [37]. Then, we looked at the size of each trajectory group. Each group should contain at least $5 \%$ of children [38]. At last, we inspected the posterior probabilities, which indicate the reliability of each trajectory classification, minimum 
threshold of 0.7 [38]. Visual exploration of the data was used to judge the adequacy of the final predicted trajectories against the actual data. To take into account the uncertainty of class trajectory assignment, the posterior classification probabilities of class membership were used as weights (same procedure as in Henselmans et al. [10]). Then, we explored the relation between adjustment trajectories and demographic, medical, and psychosocial factors. For categorical variables, chisquare tests were used, and continuous variables were tested with analyses of variance. Variables that were significantly related $(p<.05)$ to adjustment trajectories were entered in a final multinomial regression analysis. Post hoc power analysis showed that with the study sample $(N=108$, power $=.80$, alpha $=.05)$, we were able to detect medium effect sizes $\left(f^{2}=0.15\right) . P$ values (two-sided tests) $\leq 0.05$ were considered statistically significant. Cohen's $d$ was calculated as a measure for effect size. Effect sizes .20 were considered small, .50 medium, and .80 large [39].

\section{Results}

\section{Sample characteristics}

During the study period, a total of 164 families were eligible and could be invited to take part in the study of which 159 agreed (reason for rejection was feeling too overwhelmed $n=5$ ). One hundred thirty-one parents returned completed questionnaires at baseline (response rate $80 \%$ ). One patient was excluded from analyses because the questionnaire was returned long after the induction phase. Only families with complete data at two out of the three assessment moments were included in analyses $(n=108)$ of which 84 families completed all assessments. No differences were found between participants $(N=108)$ and drop-outs $(N=23)$ with respect to age $(p=.52)$ and treatment protocol $(p=.10)$. Drop-outs were more often boys $(p=.00)$. See Fig. 1 for flowchart and Table 1 for demographic information. Parentproxy measurements were performed three times for the children treated according to the standard-risk (SR) or mediumrisk (MR) ALL-10 protocol: during induction treatment (TO: $n=95, M=42.1$ days, range $=5-131$ days), after ending induction/consolidation treatment, and during maintenance treatment (T1: $n=91, M=397.4$ days, range $=348-687$ days), and shortly after finishing treatment ( $T 2: n=87$, $M=781.2$ days, range $=651-1000$ days). For children treated according to the HR ALL-10 protocol, measurements were performed only two times due to higher intensity and shorter duration of treatment: during induction treatment (T0 $n=12$, $M=46.0$ days, range $=12-96$ ), and shortly after finishing treatment ( $T 2 n=12, M=379.0$ days, range $=259-487$ days).

\section{Trajectories of behavioral problems}

\section{Internalizing behavioral problems}

Latent class growth modeling (LCGM) revealed that the linear three-trajectory model fitted best (see Table 2). The average posterior probabilities all exceeded $.70(.95, .86$, and .96$)$ and each trajectory was composed of at least $5 \%(60,30$, and

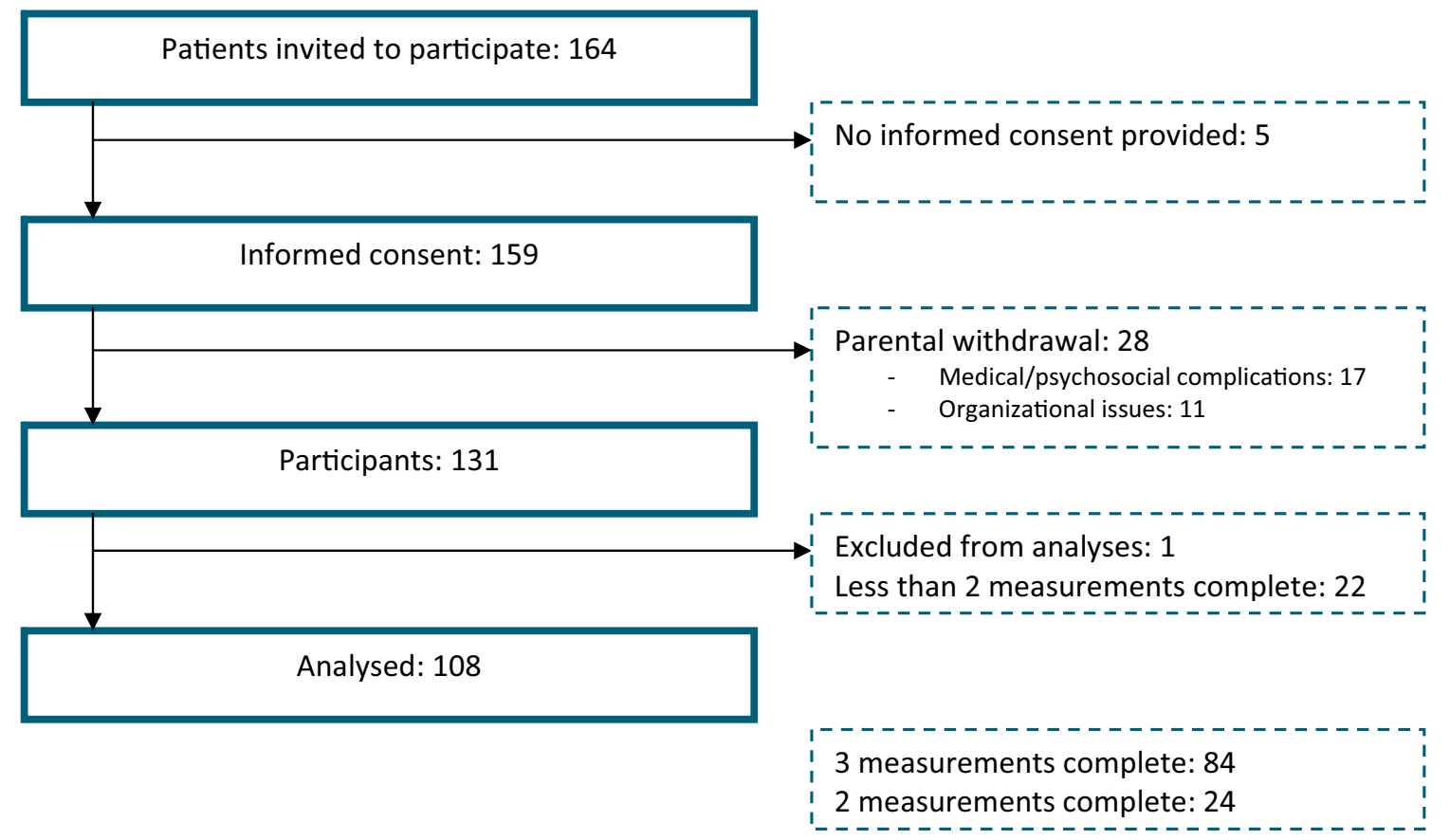

Fig. 1 Study participants and non-participants 
Table 1 Demographic information

\begin{tabular}{|c|c|}
\hline Variable & \\
\hline \multicolumn{2}{|l|}{ Age child } \\
\hline$M(\mathrm{SD})$ & $6.3(4.2)$ \\
\hline Range & $1-17$ \\
\hline \multicolumn{2}{|l|}{ Gender child } \\
\hline Male & $51(47 \%)$ \\
\hline Female & $57(53 \%)$ \\
\hline \multicolumn{2}{|l|}{ ALL treatment protocol } \\
\hline SR & $27(25 \%)$ \\
\hline MR & $69(64 \%)$ \\
\hline HR & $12(11 \%)$ \\
\hline \multicolumn{2}{|l|}{ Hospitalizations (days) } \\
\hline$M(\mathrm{SD})$ & $12.6(34.7)$ \\
\hline \multicolumn{2}{|l|}{ Range } \\
\hline ICU admissions (days) & $0-250$ \\
\hline$M(\mathrm{SD})$ & $1.7(9.5)$ \\
\hline Range & $0-76$ \\
\hline \multicolumn{2}{|l|}{ Age parent } \\
\hline$M(\mathrm{SD})$ & $38.2(5.6)$ \\
\hline Range & $25-52$ \\
\hline \multicolumn{2}{|l|}{ Gender respondent } \\
\hline Male & $15(14 \%)$ \\
\hline Female & $93(86 \%)$ \\
\hline \multicolumn{2}{|l|}{ Education level } \\
\hline Low & $13(12 \%)$ \\
\hline Medium & $51(47 \%)$ \\
\hline High & $44(41 \%)$ \\
\hline Single-parent household & $11(10 \%)$ \\
\hline
\end{tabular}

$10 \%$ respectively). The three different behavioral adjustment trajectories will now be described. Children in the resilience trajectory $(60 \%)$ did not experience any internalizing behavioral adjustment problems after diagnosis. Children in the recovery trajectory (30\%) started out with sub-clinical levels of internalizing behavioral adjustment problems but showed recovery. Children in the chronic trajectory (10\%) started out with high levels (above cut-off of 3 ) of internalizing behavioral adjustment problems, which stayed at stable heightened levels halfway through treatment. There was no recovery at the end of treatment (Fig. 2).

\section{Externalizing behavioral problems}

Table 2 shows the BIC, AIC, and estimated probabilities of the tested models. The BIC indicated that the linear two-trajectory model fitted best, whereas the AIC favored the linear threetrajectory model or the quadratic four-group model. We chose to maintain the linear three-trajectory model for several reasons. The additional third group was clearly different from the pattern showed by the two-trajectory model. It showed a chronic problem trajectory. The four-group model was not chosen, due to the significant difference in BIC of 10 . The average posterior probabilities of the linear three-trajectory model all exceeded $.70(.97, .78$, and .96$)$ and each trajectory was composed of at least $5 \%(83,12$, and $5 \%$ respectively). Children in the resilience trajectory $(83 \%)$ did not experience any externalizing behavioral adjustment problems after diagnosis. Children in the chronic trajectory (12\%) started out with clinical levels of externalizing behavioral adjustment

Table 2 Model selection results

\begin{tabular}{|c|c|c|c|c|c|c|}
\hline \multirow[t]{2}{*}{ No. of groups } & \multirow[t]{2}{*}{$\mathrm{BIC}$} & \multirow[t]{2}{*}{ AIC } & \multicolumn{4}{|c|}{ Estimated probabilities ( $\%$ in each group) } \\
\hline & & & 1 & 2 & 3 & 4 \\
\hline 1 INT linear & 1280.67 & 1267.26 & 100 & & & \\
\hline 2 INT linear & 1267.72 & 1246.27 & 86 & 14 & & \\
\hline 3 INT linear & 1260.80 & 1231.30 & 60 & 30 & 10 & \\
\hline 4 INT linear & 1274.85 & 1237.30 & 56 & 33 & 10 & 0 \\
\hline 1 EXT linear & 1368.35 & 1354.94 & 100 & & & \\
\hline 2 EXT linear & 1346.48 & 1325.02 & 90 & 10 & & \\
\hline 3 EXT linear & 1348.01 & 1318.51 & 83 & 12 & 5 & \\
\hline 4 EXT linear & 1362.06 & 1324.51 & 83 & 12 & 5 & 0 \\
\hline 1 INT quadratic & 1289.94 & 1271.17 & 100 & & & \\
\hline 2 INT quadratic & 1280.55 & 1251.05 & 89 & 11 & & \\
\hline 3 INT quadratic & 1276.58 & 1236.35 & 61 & 29 & 10 & \\
\hline 4 INT quadratic & 1287.1 & 1236.14 & 73 & 17 & 10 & 0 \\
\hline $1 \mathrm{EXT}$ quadratic & 1372.81 & 1354.04 & 100 & & & \\
\hline 2 EXT quadratic & 1354.61 & 1325.11 & 90 & 10 & & \\
\hline 3 EXT quadratic & 1361.25 & 1321.01 & 85 & 11 & 4 & \\
\hline 4 EXT quadratic & 1356.05 & 1305.09 & 83 & 6 & 6 & 5 \\
\hline
\end{tabular}

BIC Bayesian information criterion, AIC Akaike information criterion 


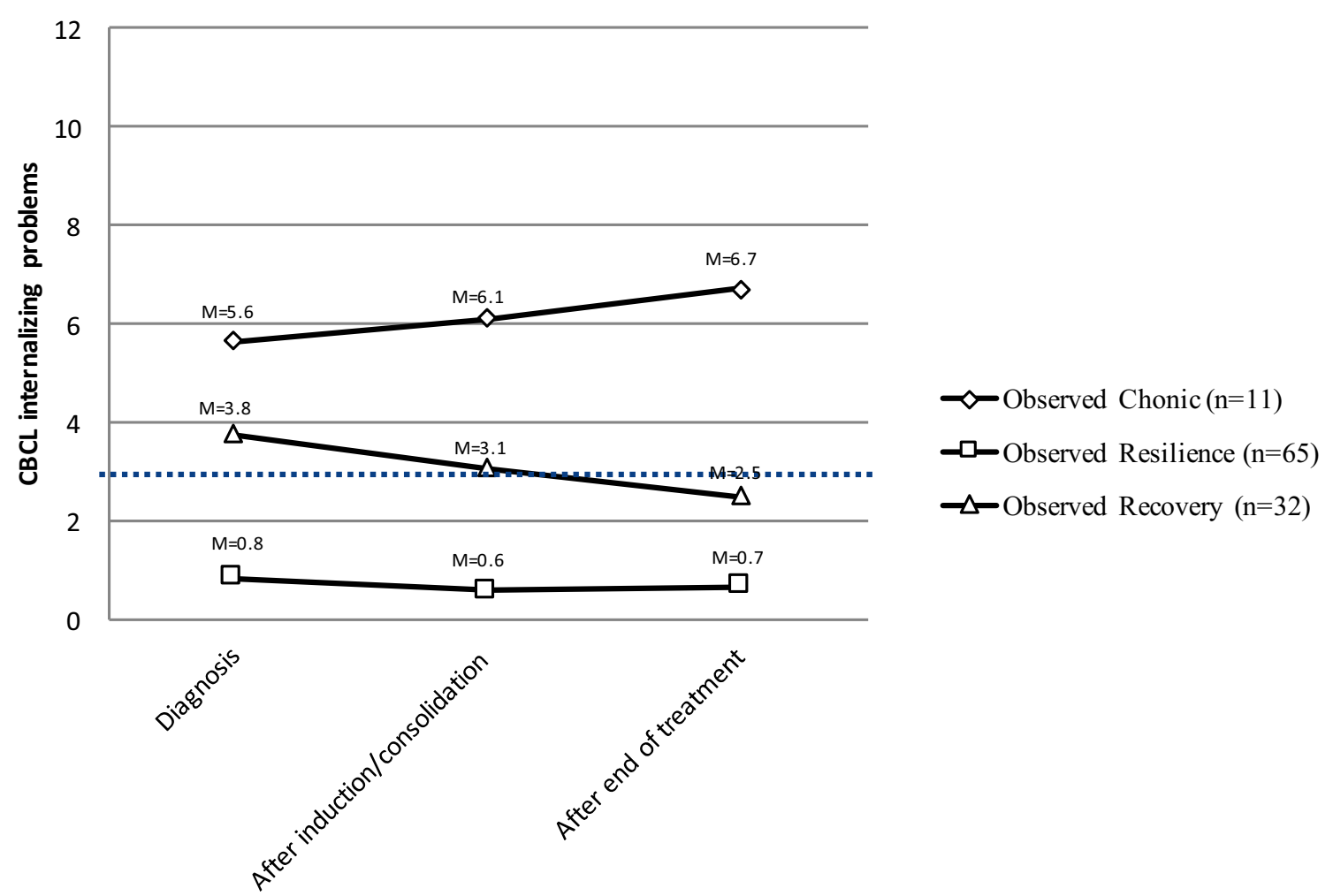

Fig. 2 Trajectories of internalizing behavioral problems. The dashed lines represent the cut-off (3) scores for which equal or higher scores indicate clinically significant symptoms

problems, which stayed stable. Children in the increasing trajectory $(5 \%)$ started out with clinical levels of externalizing behavioral problems, and showed no recovery but increasing problems during treatment (Fig. 3).

\section{Demographic, medical, and psychosocial predictors of trajectories}

\section{Internalizing behavior problems}

Table 3 shows the results of continuous and categorical predictor variables for each trajectory. Only significant predictors were entered in the final multivariate analysis. Demographic and medical characteristics were not found to be of significant influence on the trajectories. Of the personal characteristics of the parents, coping and parenting stress had a significant impact on adjustment trajectory of the child. Compared with resilience, children in the chronic trajectory had parents who showed higher levels of parenting stress $(p<.001 ; d=1.20)$ after the cancer diagnosis. At last, child physical and psychological QoL at diagnosis were significantly associated with trajectory membership. Compared with the resilience trajectory, children in the chronic trajectory experienced lower physical QoL $(p<.01 ; d=.94)$, psychosocial QoL $(p<.001 ; d=1.53)$, and cancer-related QoL $(p<.001$; $d=1.51)$ at diagnosis.

\section{Externalizing behavior problems}

Only parenting stress had a significant impact on adjustment trajectory of the child. Compared with resilience, children in the increasing trajectory had parents who showed higher levels of parenting stress $(p<.001 ; d=2.30)$ after the cancer diagnosis. Also, children in the chronic trajectory had parents experiencing more parenting stress $(p<.01 ; d=.91)$.

\section{Multivariate regression analyses}

For internalizing problems, the personal characteristics of the parents (parenting stress) and baseline adaptation of the child (physical, psychosocial, and cancer-related QoL) were entered simultaneously in a multinomial logistic regression analysis with the Resilience trajectory as the reference group (Table 4). The final model was statistically significant $\left(\mathrm{X}^{2}=58.906\right.$, $d f=8, p<.001$, Cox \& Snell $\mathrm{R}^{2}=0.45$, Nagelkerke $=0.54$, McFadden $=0.34)$. Only parenting stress $\left(\chi^{2}=11.02, p<.01\right)$ and baseline cancer-related QoL of the child $\left(\chi^{2}=18.08\right.$, $p<.001)$ were still a significant predictor of behavioral adjustment trajectory. Children with parents experiencing higher levels of parenting stress (recovery odds ratio $=1.01$; chronic odds ratio $=1.10$ ) and lower baseline cancer-related QoL (recovery odds ratio $=.93$; chronic odds ratio $=.83$ ) were more likely to belong to the recovery or chronic trajectory than to 


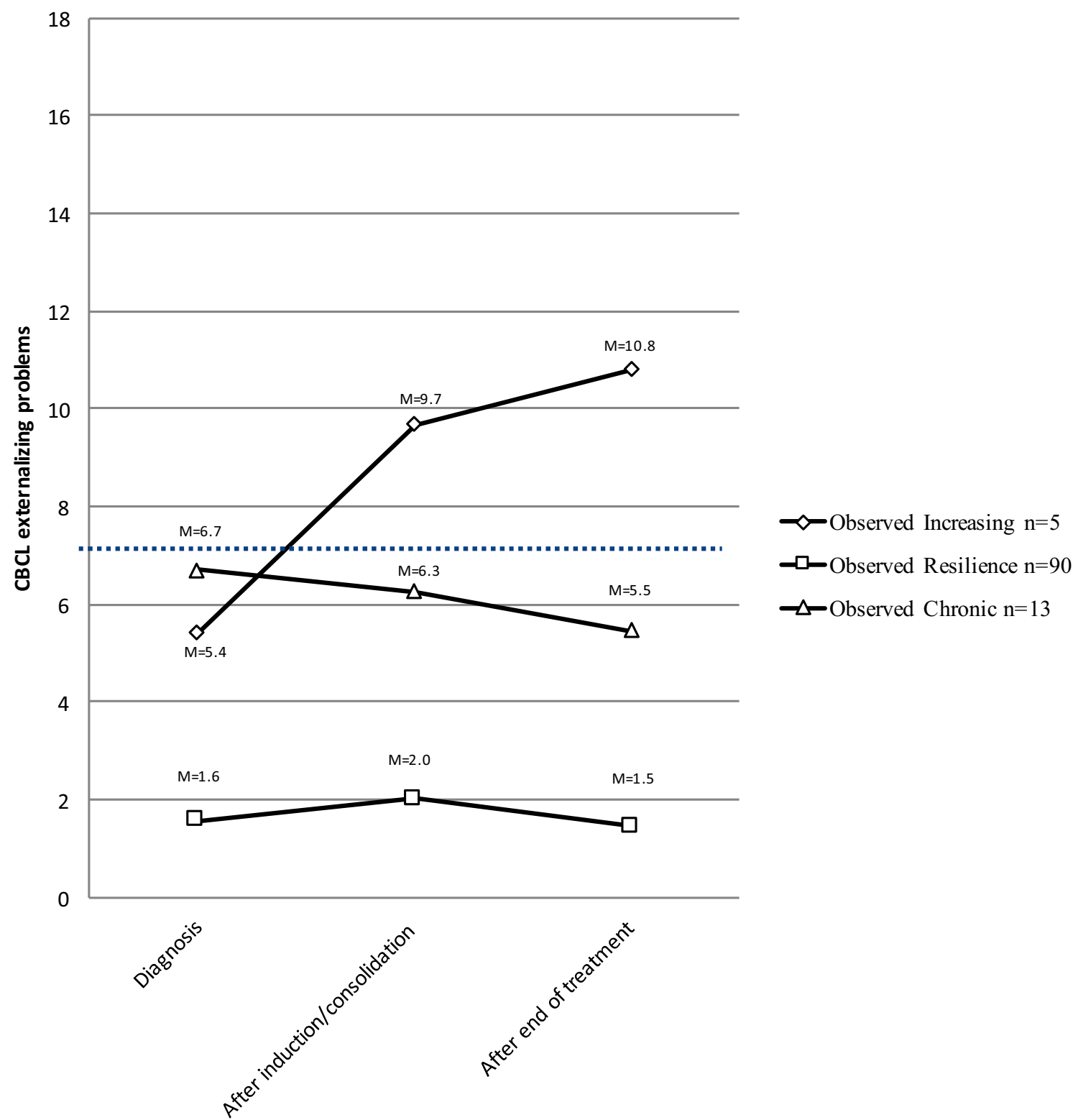

Fig. 3 Trajectories of externalizing behavioral problems. The dashed lines represent the cut-off (5) scores for which equal or higher scores indicate clinically significant symptoms

the resilience trajectory. For externalizing problems, only parenting stress was entered into the regression analyses (Table 4). The final model was statistically significant $\left(X^{2}=21.289, d f=2, p<.001\right.$, Cox \& Snell $R^{2}=0.19$, Nagelkerke $=0.30$, McFadden $=0.21$ ). Children with a parent who experienced higher levels of parenting stress were more likely to belong to the increasing (odds ratio $=1.12$ ) or chronic trajectory (odds ratio $=1.05$ ) than to the resilience trajectory.

\section{Discussion}

This study identified three distinctive trajectories of both internalizing and externalizing behavioral adjustments, comparable to previous research [10]. The majority of the children $(53 \%)$ showed no behavioral problems during the entire treatment as reported by parents. A smaller group of children $(24 \%)$ showed adjustment problems at diagnosis, but recovered at the end of treatment to normal. Adjustment problems that not returned to normal were present in a substantial group of children (19\%). Severe maladjustment was present in a small but substantial group of the patients (4\%), experiencing high levels of both internalizing and externalizing behavioral problems. Thus, most children diagnosed with ALL seem to adjust relatively well regarding their psychosocial well-being.

The categorization into these groups is in concordance with the Psychosocial Preventative Health Model (PPPHM) as 


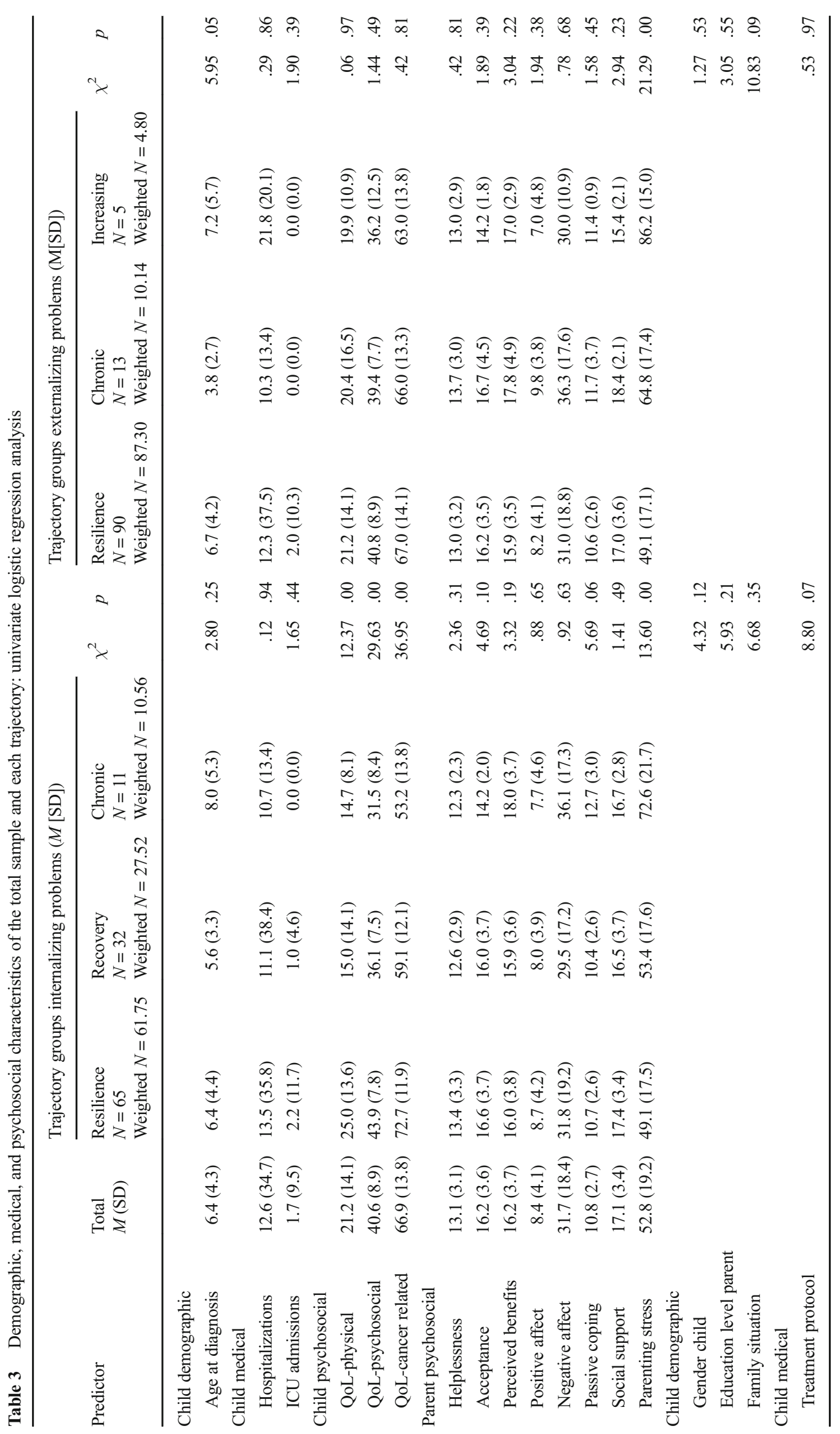


Table 4 Multinomial regression analysis results for internalizing (chronic and recovery versus resilience trajectory) and externalizing (increasing and chronic versus resilience trajectory) behavior problems

\begin{tabular}{llllll}
\hline & $\chi^{2}$ & $p$ & $B$ & Wald & Exp $(B)$ \\
\hline $\begin{array}{l}\text { Predictor internalizing } \\
\text { Parenting stress }\end{array}$ & 11.02 & .00 & & & \\
$\quad$ Recovery & & & .01 & 0.62 & 1.01 \\
$\quad$ Chronic & & & .09 & 7.84 & 1.10 \\
QoL-physical & 2.67 & .26 & & & \\
$\quad$ Recovery & & & -.03 & 1.88 & .97 \\
$\quad$ Chronic & & & -.05 & 1.52 & .95 \\
QoL-psychosocial & 3.82 & .15 & & & \\
$\quad$ Recovery & & & -.07 & 3.52 & .93 \\
$\quad$ Chronic & & & -.07 & 0.98 & .93 \\
QoL-cancer related & 18.08 & .00 & & & \\
$\quad$ Recovery & & & -.08 & 9.17 & .93 \\
Chronic & & & -.18 & 8.46 & .83 \\
Predictor externalizing & & & & & \\
Parenting stress & 21.29 & .00 & & & \\
$\quad$ Increasing & & & .11 & 9.64 & 1.12 \\
Chronic & & & .05 & 6.30 & 1.05 \\
\hline
\end{tabular}

described by Kazak [40]. Although this model is focused on family adjustment, it might also be applied to child adaptation due to the important influence of the family on the ill child. Compared to research in adults, children seem to have a more resilient trajectory of adjustment [10]. However, we know that a substantial amount of the survivors of childhood cancer experience ongoing problems even long time after the ending of treatment, apparently more than children during active treatment. The adequate adjustment of children during treatment seems therefore treacherous: during the structured period of treatment, children are adapting quite well, but after the end of treatment, a period in which the number of hospital visits declines, a growing number of children experience late effects. Psychosocial care is important for these groups of patients, to support the process of getting back to life as usual.

This study also showed that not medical factors, such as diagnosis and number of hospitalizations, of the child put them at risk for psychosocial difficulties, but mainly the psychological reaction of the parents after diagnosis. Children with chronic high scores regarding internalizing behavior could be distinguished from the group that showed recovery by more passive coping by the parents, and higher levels of parenting stress. Children with growing externalizing problems could be distinguished from the stable group by higher levels of parenting stress. A link between passive coping style and adverse psychological reactions has been reported repeatedly before in both pediatric and adult care $[19,41]$. Therefore, it can be seen as a substantial risk factor for maladjustment after diagnosis and should be paid attention to. At last, an effect for parenting stress has also been previously reported, with higher levels of stress being indicative for family adjustment difficulties [21]. Attention is needed for families experiencing parenting stress while raising a child with a chronic and life-threatening illness. Interventions focused on reducing stress, for example by improving problem solving skills, would be helpful for this specific vulnerable population.

As mentioned before, this study did not find an effect for demographic and medical variables of the patient and on behavioral adjustment problems. This lack of effect of gender [7] and age at diagnosis [42] on behavioral problems has been previously reported. An effect for medical risk stratification has been previously reported, with higher treatment intensity being indicative for behavioral problems [2]. However, differences in time since diagnosis and treatment protocol limit comparisons. Also, illness cognitions, parental affect, and social support were not found to be of significant influence. It might be that these factors are mainly associated with parental distress and not directly influence behavior of the child after diagnosis [19, 25].

This study with its longitudinal design and a homogenous population made it possible to investigate patterns of behavioral adjustment after the diagnosis of childhood cancer. Instead of examining this at group level, we looked at individual differences in trajectories of behavioral adaptation. By aggregating these individual differences, we found three distinctive patterns of behavioral adjustment problems over time, for both internalizing and externalizing symptoms. This approach provided us with more advanced knowledge about the course of adjustment in children diagnosed with cancer. However, there are also some limitations. This study included child behavior problems and parenting stress as important variables. Parenting stress was treated as predictor and behavior problems as the outcome, based on previous findings. However, they also might influence each other the other way around: child behavior might lead to parenting stress. The longitudinal nature of this study provided evidence for the framework we tested; however, the effect of behavior problems on parenting stress could not be delineated with the current study. Next to this, the power to detect differences between groups was limited due to little $N$ in the smallest internalizing and eternalizing classes. Furthermore, we focused on the behavioral adjustment during treatment, which might limit the ability to draw conclusions on long-term behavioral adjustment. Further studies need to be performed with a longer follow-up period and broader patient sample to evaluate the course of behavioral adjustment of children with cancer in general into long-term survivorship. In addition, we only used parent-proxy reports in this study due to the young median age of children diagnosed with ALL. Parent reports were used to assess both parent and child functioning, and from previous research, it is known that child behavior judged by parents is difficult to interpret [43]. At last, selection bias might be present in this study 
sample. It could be that the parents who dropped out of the study were more stressed or had children with greater problems compared to those who completed all assessment time points.

The results of this study show the importance of early identification of patients at risk for ongoing or escalating problems. Screening would facilitate this, assessing a broad range of risk factors, such as child baseline adaptation, parental stress, and coping. Information on such risk and protective factors is helpful to provide personalized, family-based, and cost-effective psychosocial care [40].

Compliance with ethical standards The study was approved by each of the medical ethical review boards of the participating institutions. Informed consent was obtained from all participants in this study.

Conflict of interest This study was supported by the Dutch Childhood Oncology Group (SKION). The authors have had full access to all data in the study and had full responsibility for the design of the study, collection, analysis, and interpretation of data, and the writing of the report.

Open Access This article is distributed under the terms of the Creative Commons Attribution-NonCommercial 4.0 International License (http://creativecommons.org/licenses/by-nc/4.0/), which permits any noncommercial use, distribution, and reproduction in any medium, provided you give appropriate credit to the original author(s) and the source, provide a link to the Creative Commons license, and indicate if changes were made.

\section{References}

1. Imbach P, Kühne T, Arceci R (eds) (2006) Pediatric oncology: a comprehensive guide. Springer, Berlin, pp. 5-28

2. Buizer AI, De Sonneville LMJ, Van Den Heuvel-Eibrink MM, et al. (2006) Behavioral and educational limitations after chemotherapy for childhood acute lymphoblastic leukemia or Wilms tumour. Cancer 106:2067-2075. doi:10.1002/cncr.21820

3. Reinfjell T, Lofstad GE, Nordahl HM, et al. (2009) Children in remission from acute lymphoblastic leukeamia: mental health, psychosocial adjustment and parental functioning. Eur J Cancer Care 18:364-370. doi:10.1111/j.1365-2354.2008.00954.x

4. Krull KR, Brinkamn TM, Li C, et al. (2013) Neurocognitive outcomes decades after treatment for childhood acute lymphoblastic leukemia: a report from the St Jude Lifetime Cohort Study. J Clin Oncol 31(35):4407-4415. doi:10.1200/JCO.2012.48.2315

5. Shah NN, Wayne AS (2015) Leukemias and lymphomas. In: Wiener L, Pao M, Kazak AE, Kupst M, Farkas Patenaude A, Arceci RJ (eds) Pediatric psycho-oncology, 2nd edn. Oxford University Press, New York, pp. 3-9

6. Tsai M, Hsu J, Chou W, et al. (2013) Psychosocial and emotional adjustment for children with pediatric cancer and their primary caregivers and the impact on their health-related quality of life during the first 6 months. Qual Life Res 22:625-634. doi:10. 1007/s11136-012-0176-9

7. Marcoux S, Robaey P, Krajinovic M, et al. (2012) Predictive factors of internalized and externalized behavioral problems in children treated for acute lymphoblastic leukemia. Pediatr Blood Cancer 58:971-977. doi: $10.1002 / p b c .24079$

8. Noll RB, Gartstein MA, Vannatta K, et al. (1999) Social, emotional, and behavioral functioning of children with cancer. Pediatrics 103:71-78
9. Dunn J, Ng SK, Holland J, et al. (2013) Trajectories of psychological distress after colorectal cancer. Psycho-Oncology 22:17591765. doi:10.1002/pon. 3210

10. Henselmans I, Seltman H, Helgeson VS, De Vries J, Sanderman R, Ranchor AV (2010) Identification and prediction of distress trajectories in the first year after a breast cancer diagnosis. Health Psychol 29(2):160-168. doi:10.1037/a0017806

11. Boyes AW, Girgis A, D'Este CA, et al. (2013) Prevalence and predictors of the short-term trajectory of anxiety and depression in the first year after a cancer diagnosis: a population-based longitudinal study. J Clin Oncol 31:2724-2729. doi:10.1200/JCO.2012. 44.7540

12. Kazak AE, Schneider S, Kassam-Adams N (2009) Pediatric medical traumatic stress. In: Roberts MC, Steele RG (eds) Handbook of pediatric psychology, 4th edn. The Guilford Press, New York, pp. 205-215

13. Brinkman TM, Palmer SL, Chen S, et al. (2012) Parent-reported social outcomes after treatment for pediatric embryonal tumors: a prospective longitudinal study. J Clin Oncol 30(33):4134-4140. doi:10.1200/JCO.2011.40.6702

14. Kullgren KA, Morris RD, Morris MK, Krawiecki N (2003) Risk factors associated with long-term social and behavioral problems among children with brain tumors. J Psychosoc Oncol 21(1):73-87. doi:10.1300/J077v21n01 04

15. Penn A, Shortman RI, Lowis SP, et al. (2010) Child-related determinants of health-related quality of life in children with brain tumours 1 year after diagnosis. Pediatr Blood Cancer 55:1377-1385. doi: $10.1002 / p b c .22743$

16. Maurice-Stam H, Oort FJ, Last BF, Brons PPT, Caron HN, Grootenhuis MA (2008) Longitudinal assessment of healthrelated quality of life in preschool children with non-CNS cancer after the end of successful treatment. Pediatr Blood Cancer 50: 1047-1051. doi:10.1002/pbc.21374

17. Frank NC, Brown RT, Blount RL, Bunke V (2001) Predictors of affective responses of mothers and fathers of children with cancer. Psycho-Oncology 10(4):293-304. doi:10.1002/pon.526

18. Stuber ML, Kazak AE, Meeske K, et al. (1997) Predictors of posttraumatic stress symptoms in childhood cancer survivors. Pediatrics 100:958-964. doi:10.1542/peds.100.6.958

19. Grootenhuis MA, Last BF (1997) Predictors of parental emotional adjustment to childhood cancer. Psycho-Oncology 6:115-128

20. Fedele DA, Mullins LL, Wolfe-Christensen C, Carpentier MY (2011) Longitudinal assessment of maternal parenting capacity variables and child adjustment outcomes in pediatric cancer. J Pediatr Hematol Oncol 33(3):199-202

21. van der Geest IM, van den Heuvel-Eibrink MM, Passchier J, et al. (2014) Parenting stress as a mediator of parents' negative mood state and behavior problems in children with newly diagnosed cancer. Psycho-Oncology 23(7):758-765. doi:10. 1002/pon.3475

22. Van Tilburg CM, Sanders EAM, Nibbelke EE, et al. (2011) Impact of reduced chemotherapy treatment for good risk childhood acute lymphoblastic leukaemia on infectious morbidity. Br J Haematol 152:433-440. doi:10.1111/j.1365-2141.2010.08463.x

23. Wald FDM, Mellenbergh GJ (1990) De verkorte versie van de Nederlandse vertaling van de profile of moods states (POMS). Nederlands tijdschrift voor de Psychologie 45:86-90

24. McNair DM, Lorr M, Droppleman LF (1971) Edits manual for the profile of mood states. Educational and Industrial Testing Service, San Diego, Cal.

25. Sint Nicolaas SM, Schepers SA, Meijer-van den Bergh EMM, Evers AWM, Hoogerbrugge PM, Grootenhuis MA, Verhaak CM (2015) Illness cognitions and family adjustment: psychometric properties of the Illness Cognition Questionnaire in parents of a child with cancer. Support Care Cancer. doi:10.1007/s00520-015-2795-5 
26. Evers AWM, Kraaimaat FW, Van Lankveld W, Jacobs JWG, Bijlsma JWJ (1998) De ziekte-cognitie-lijst (ZCL). Gedragstherapie 31:205-220

27. Schreurs PJG, Van de Willige G, Brosschot JF, Tellegen B, Graus GMH (1993) Handleiding Utrechtse coping lijst UCL (herziene druk). Swets \& Zeitlinger, Lisse

28. De Brock AJLL, Vermulst AA, Gerris JRM, Abidin RR (1992) NOSI, Nijmeegse ouderlijke stress index. Swets \& Zeitlinger, Lisse

29. Van Dam-Baggen R, Kraaimaat F (1992) De inventarisatielijst sociale betrokkenheid (ISB): Een zelfbeoordelingslijst om sociale steun te meten. Gedragstherapie 25:27-46

30. Raat H, Bonsel GJ, Essink-Bot ML, Landgraf JM, Gemke RJBJ (2002) Reliability and validity of comprehensive health status measures in children: the Child Health Questionnaire in relation to the Health Utilities Index. J Clin Epidemiol 55(1):67-76

31. Varni JW, Burwinkle TM, Katz ER, Meeske K, Dickinson P (2002) The PedsQ1 in pediatric cancer: reliability and validity of the pediatric quality of life inventory generic core scales, multidimensional fatigue scale, and cancer module. Cancer 94(7):2090-2106

32. Achenbach TM (ed) (1991) Manual for the child behavior checklist 4-18 and 1991 profiles. University of Vermont Department of Psychiatry, Burlington

33. Drotar D, Stein REK, Perrin EC (1995) Methodological issues using the child behavior checklist and its related instruments in clinical child psychology research. J Clin Child Psychol 24:184-192

34. Gilliom M, Shaw DS (2004) Codevelopment of externalizing and internalizing problems in early childhood. Dev Psychopathol 16: 313-333
35. Van der Ende J, Verhulst FC, Tiemeier H (2012) Agreement of informants on emotional and behavioral problems from childhood to adulthood. Psychol Assess 24(2):293-300

36. Proust-Lima, C. and B. Liquet (2009) LCMM: an R package for estimation of latent class mixed models and joint latent class models, R, cran

37. Airila A, Hakanen JJ, Luukkonen R, et al. (2014) Developmental trajectories of multisite musculoskeletal pain and depressive symptoms: the effects of job demands and resources and individual factors. Psychol Health 29(12):1421-1441

38. Nagin DS, Odgers CL (2010) Group-based trajectory modeling in clinical research. Annu Rev Clin Psychol 6:109-138

39. Cohen JA (1992) Power primer. Psychol Bull 112:155-159

40. Kazak AE (2006) Pediatric psychosocial preventative health model (PPPHM): research, practice, and collaboration in pediatric family systems medicine. Fam Syst Health 24(4): 381-395

41. Pieterse K, van Dooren S, Seynaeve C, et al. (2007) Passive coping and psychological distress in women adhering to regular breast cancer surveillance. Psycho-Oncology 16:851-858

42. Landolt MA, Ystrom E, Sennhauser FH, Gnehm HE, Vollrath ME (2012) The mutual prospective influence of child and parental posttraumatic stress symptoms in pediatric patients. J Child Psychol Psychiatry 53(7):767-777

43. Eiser C, Morse R (2001) Can parents rate their child's health-related quality of life? Results of a systematic review. Qual Life Res 10: $347-357$ 